\title{
Density Matrix Renormalization Group Numerical Study of the Kagome Antiferromagnet
}

\author{
H. C. Jiang, ${ }^{1,2}$ Z. Y. Weng, ${ }^{1}$ and D. N. Sheng ${ }^{2}$ \\ ${ }^{1}$ Center for Advanced Study, Tsinghua University, Beijing, 100084, China \\ ${ }^{2}$ Department of Physics and Astronomy, California State University, Northridge, California 91330, USA \\ (Received 15 April 2008; revised manuscript received 13 August 2008; published 10 September 2008)
}

\begin{abstract}
We numerically study the spin- $\frac{1}{2}$ antiferromagnetic Heisenberg model on the kagome lattice using the density-matrix renormalization group method. We find that the ground state is a magnetically disordered spin liquid, characterized by an exponential decay of spin-spin correlation function in real space and a magnetic structure factor showing system-size independent peaks at commensurate magnetic wave vectors. We obtain a spin triplet excitation gap $\Delta E(S=1)=0.055 \pm 0.005$ by extrapolation based on the large size results, and confirm the presence of gapless singlet excitations. The physical nature of such an exotic spin liquid is also discussed.
\end{abstract}

DOI: 10.1103/PhysRevLett.101.117203

PACS numbers: 75.10.Jm, 75.40.Mg, 75.50.Ee

Novel magnetic properties and the possible existence of exotic spin-liquid states [1] in low-dimensional spin- $\frac{1}{2}$ systems have attracted intensive attention in recent years. It has been established that spins in the ground state of the two dimensional (2D) nearest-neighbor Heisenberg antiferromagnet (HAF) model

$$
H=\sum_{\langle i, j\rangle} \mathbf{S}_{i} \cdot \mathbf{S}_{j},
$$

are still ordered on square [2] and triangular [3,4] lattice systems. However, spin-liquid states are likely to be found in some geometrically more frustrated systems [5], like the kagome lattice, which may be seen as a diluted triangular lattice (see Fig. 1) with larger geometrical frustration and lower coordination number than the triangular lattice. Earlier exact diagonalization (ED) studies [6-8] suggest that the kagome antiferromagnet has a short-range spin correlation and a possible finite spin gap $\sim 0.05$ when the finite-size results (up to $N=36$ sites) are extrapolated to the thermodynamic limit. Within the spin gap, a large number of singlet excited states are also identified $[8,9]$. Recently, algebraic vortex liquid and Dirac spin liquid with gapless Dirac fermion excitations have been also proposed $[10,11]$. Such a Dirac spin-liquid state has a reasonably good variational energy [11], but the vanishing spin triplet gap is in contrast to the ED result. While the discrepancy may be attributed [11] to the uncertainty of the finite-size effect in the ED, alternatively a finite spin gap can be also gained in the Dirac spin-liquid state via an instability [12] towards a valence bond crystal (VBC) state with a broken translational symmetry. Earlier on, Zeng and Marston [13] also proposed that the ground state of the kagome HAF appears to be a VBC state with a 36-site unit cell, which is supported by the series expansions [14]. So far the precise nature of the HAF on the kagome lattice in the longwavelength and low-energy regime remains unsettled.

Experimentally the newly synthesized Herbertsmithite $\mathrm{ZnCu}_{3}(\mathrm{OH})_{6} \mathrm{C}_{12}$, in which the spin- $\frac{1}{2}$ copper ions form layered kagome lattices, has brought tremendous excitement to this field. The absence of the magnetic ordering has been established based on the neutron scattering measurement [15] down to $50 \mathrm{mK}$, as compared to a relatively high Curie-Weiss temperature $(\sim 300 \mathrm{~K})$. The magnetic measurements [15-18] also suggest that there is no signature of a finite spin gap seen in the experiment, which seems consistent with an algebraic spin liquid, but contrary to a short-range spin-liquid state with a finite triplet gap. However, possible impurity spins outside the kagome layers, caused by substitutions of nonmagnetic $\mathrm{Zn}$ sites with $\mathrm{Cu}$, or the presence of Dzyaloshinsky-Moriya interactions [19], may all play an important role in order to fully understand the experimental results. While the experimental situation is still unclear, on the fundamental side, it is highly desirable to reexamine the issues regarding the nature of the ground state and low-lying excitations in a pure spin- $\frac{1}{2}$ HAF model on the kagome lattice.

In this Letter, we present a systematic numerical study by employing the density-matrix renormalization group (DMRG) method [20]. We find that the ground state is indeed a magnetically disordered state, which is charac-

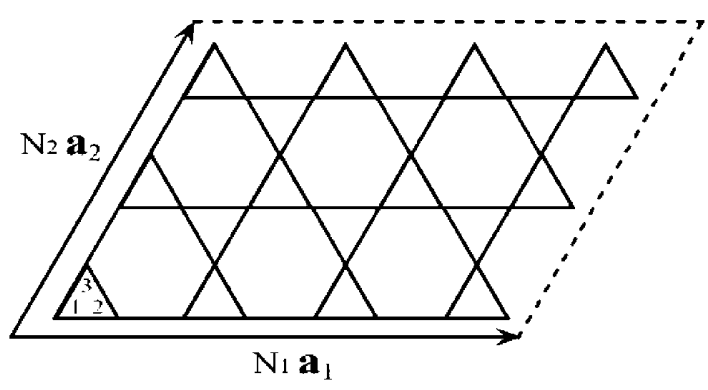

FIG. 1. Sketch of a three-leg kagome lattice with total number of sites $N=3 \times N_{1} \times N_{2}$ and number of unit cells $N_{1} \times N_{2}=$ $4 \times 3$. Here $\mathbf{a}_{1}=(2,0)$ and $\mathbf{a}_{2}=(1, \sqrt{3})$ are two primitive vectors of the unit cell including three inequivalent sites (e.g., $1,2,3)$ 
terized by an exponential decay of the equal-time spin-spin correlation function in real space. The corresponding magnetic structure factor shows small peaks at commensurate momenta, with near constant peak values insensitive to the size of the system, in sharp contrast to the structure factor of the magnetic ordered state on a triangular lattice. Furthermore, we calculate the spin triplet gap, which is extrapolated to a finite value $\Delta E(S=1)=0.055 \pm 0.005$ in the large sample size limit. In this spin-liquid state, there also exist low-lying singlet excitations, with their gap approaching zero at the large sample size limit. Our calculations strongly hint that the ground state may be described by a resonating valence bond (RVB) spin liquid with short-range spin and dimer-dimer correlations without explicitly breaking lattice symmetries.

We consider a kagome lattice with finite length vectors $N_{1} \mathbf{a}_{1}$ and $N_{2} \mathbf{a}_{2}$ as shown in Fig. 1. Here $\mathbf{a}_{1}=(2,0)$ and $\mathbf{a}_{2}=(1, \sqrt{3})$ are two primitive vectors of the unit cell which includes three lattice sites on a triangle. The total number of sites is $N=3 \times N_{1} \times N_{2}$, with the number of unit cells $N_{1} \times N_{2}$. We will extend the calculation from $N=36$ (the maximum size for ED) to much larger sizes with different geometries, up to $N=3 \times 16 \times 4$ (192 sites), using the DMRG method. To test the performance of the DMRG method in the 2D spin systems, we have compared our results with the ED up to $N=36$ sites for various lattices (including triangular, square, and kagome lattices) and obtained accurate ground state energies with errors smaller than $0.01 \%$. For the present study, we keep up to $m=4096$ states in the DMRG block for most systems with more than 24 sweeps to get a converged result, and the truncation error is of the order or less than $10^{-5}$. We make use of the periodic boundary condition to reduce the finite-size effect for a more reliable extrapolation to the thermodynamic limit.

We first present the DMRG result for a system with $N=$ 48 sites $\left(N_{1}=N_{2}=4\right)$. In Fig. 2(a), we show the ground state energy per site $E_{0} / N$ as a function of $m$-the number of states kept in each block (the dimension of the Hilbert space $\left.=4 m^{2}\right) . \quad E_{0} / N \quad$ is extrapolated to -0.43663 at the large $m$ limit, and the estimated error at $m=4096$ is about $0.16 \%$. Similarly, the lowest energy per site $E_{1} / N$ in the total spin $S=1$ sector is also shown in Fig. 2(a). Defining the spin triplet gap $\Delta E(S=1) \equiv$ $E_{1}(S=1)-E_{0}$, such a spin gap starts to saturate at $m>$ 2000 and approaches the value 0.145 at large $m$ as plotted in the inset of Fig. 2(a). In Fig. 2(b), similar results for a larger system with $N=108(3 \times 12 \times 3)$ are also shown, where we find a slightly larger spin gap at 0.163 for this larger but narrow system.

A systematic size dependence of the spin gap is shown in Fig. 3. In the main panel, the spin gap $\Delta E(S=1)$ (solid squares) vs $1 / N$ is plotted with $N=3 \times 4 \times 3,3 \times 6 \times 3$, $3 \times 4 \times 4,3 \times 6 \times 4,3 \times 8 \times 4,3 \times 6 \times 5$ and up to $3 \times$ $6 \times 6=108$, together with the results of the ED [7] (open

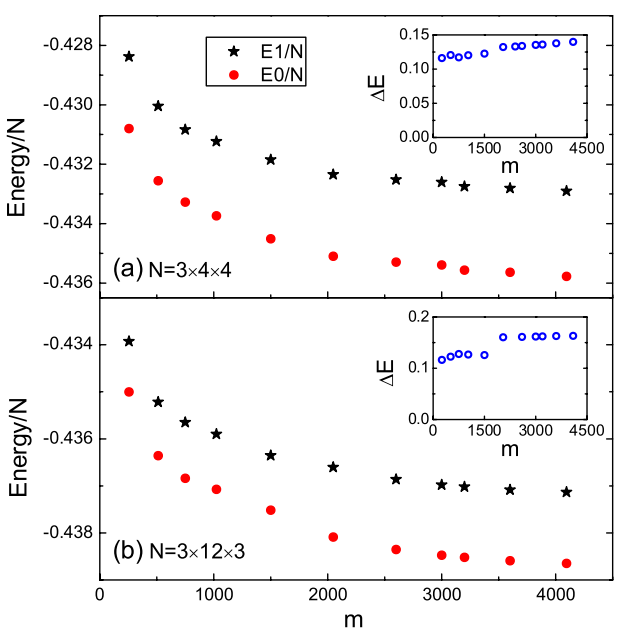

FIG. 2 (color online). (a) The ground state energy per site $E_{0} / N$ (solid circles) and the excitation energy $E_{1} / N$ (solid stars) in the total spin $S=1$ sector are shown as a function of $m$ (the number of states kept in each block) for the system with $N=$ $3 \times 4 \times 4$; (b) for a system $N=3 \times 12 \times 3$. The energy gap for spin-1 excitation $\Delta E=E_{1}-E_{0}$ (open circles) as a function of $m$ for these two systems are shown in the insets.

circles) at smaller sizes (note that $N=36$ site system in the ED has a different geometry as compared to the $N=$ $3 \times 4 \times 3$ system in the present calculation). All these data follow nicely a straight line shown in Fig. 3, which allows us to extrapolate the spin gap to a finite value $\Delta E(S=$ 1) $=0.055 \pm 0.005$ in the thermodynamic limit. Note that all the data presented in the main panel are for the systems close to squarelike with the aspect ratio $\alpha=N_{1} / N_{2}$ in the range of $1 \leq \alpha \leq 2$. The corresponding ground state energies per site $\epsilon_{0}=E_{0} / N$ and the spin gaps $\Delta E(S=1)$ for the various system sizes at a given $m=4096$ are listed in

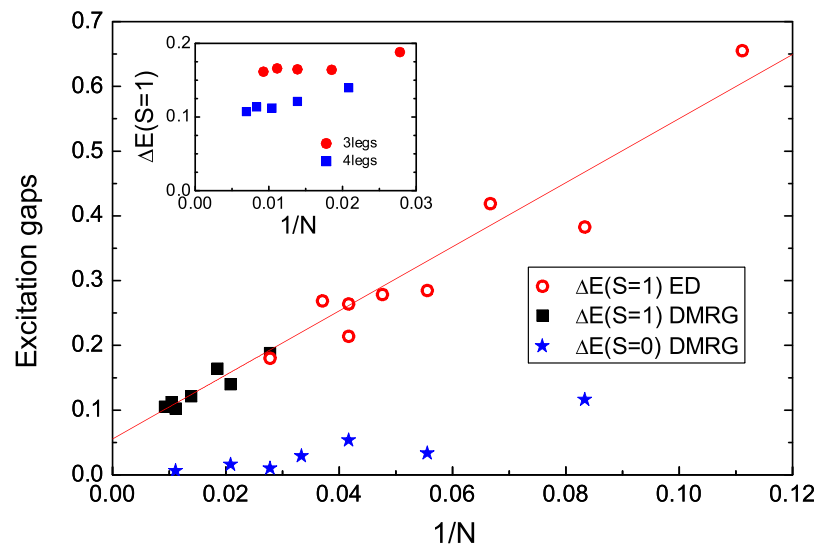

FIG. 3 (color online). The spin gap $\Delta E(S=1)$ for squarelike systems (see text) at different system sizes obtained from the ED [7] (open circles) and DMRG (solid squares) with $N=36-108$. The singlet excitation gap $\Delta E(S=0)=E_{1}(S=0)-E_{0}$ is also given (solid stars). Inset shows the spin gap for both 3-leg and 4leg systems. 
TABLE I. The ground state energy per site $\epsilon_{0}$ and spin gap $\Delta E(S=1)$ for the squarelike kagome lattice, obtained by the DMRG with keeping $m=4096$ basis states in one block. The average CPU time for each data point is about two weeks on a single Intel processor.

\begin{tabular}{lcc}
\hline \hline$N$ & $\epsilon_{0}$ & $\Delta E(S=1)$ \\
\hline $3 \times 4 \times 3$ & -0.43898 & 0.188 \\
$3 \times 6 \times 3$ & -0.43875 & 0.164 \\
$3 \times 8 \times 3$ & -0.43867 & 0.165 \\
$3 \times 10 \times 3$ & -0.43868 & 0.163 \\
$3 \times 12 \times 3$ & -0.43865 & 0.163 \\
$3 \times 4 \times 4$ & -0.43591 & 0.140 \\
$3 \times 6 \times 4$ & -0.43564 & 0.122 \\
$3 \times 8 \times 4$ & -0.43556 & 0.112 \\
$3 \times 10 \times 4$ & -0.43552 & 0.114 \\
$3 \times 6 \times 6$ & -0.43111 & 0.105 \\
\hline \hline
\end{tabular}

Table I. For the $N=3 \times 6 \times 6$ system, we keep up to $m=$ 6000 basis states and find $\epsilon_{0}=-0.43160$. For this system, $\epsilon_{0}$ as a function of $m$ extrapolates to $\epsilon_{0}=-0.4332$ at $m \rightarrow \infty$, which serves as a good estimate of the ground state energy in the thermodynamic limit [21]. Furthermore, in the inset of Fig. 3, the spin gap vs $1 / N$ for $3-\operatorname{leg}(N=$ $\left.3 \times N_{1} \times 3\right)$ and 4-leg $\left(N=3 \times N_{1} \times 4\right)$ systems with $N_{1}=4-12$ (thus including larger $\alpha$ 's) are also present for comparison. In general, the spin gap of the 4-leg systems is smaller than that of the 3-leg systems due to the finite-size effect, consistent with the behavior shown in the main panel for the more squarelike systems.

Besides the spin triplet gap $\Delta E(S=1)$, the lowest singlet excitation energy $\Delta E(S=0) \equiv E_{1}(S=0)-E_{0}$ is also shown in the main panel of Fig. 3 (solid stars), whose magnitude is much smaller than $\Delta E(S=1)$ and approaches zero with increasing sample size. This is consistent with the ED results $[8,9]$ in smaller systems, in which a large number of singlet states below the spin gap, growing with the system size, are identified. In contrast to the finite spin triplet gap, such vanishing singlet excitation energy indicates that the low-lying singlet excitations will play a dominant role in the low-temperature thermodynamic properties like specific heat.

To describe the magnetic property of the ground state, the spin-spin correlation function $\left|\left\langle S_{0}^{z} S_{r}^{z}\right\rangle\right|$ is presented in Fig. 4 for two systems with $N=3 \times 10 \times 3$ and $3 \times 10 \times$ 4 , respectively. Here $r$ is the distance between the two sites along the $\mathbf{a}_{1}$ direction in units of the lattice constant and the error bar denotes the mean square deviation for all the equivalent pairs of sites. Figure 4 shows that the results are well fitted by the straight lines representing an exponential fit: $\left|\left\langle S_{0}^{z} S_{r}^{z}\right\rangle\right|=A \exp (-r / \tau)$ with $\tau$ as the spin correlation length whose size is insensitive to the number of legs and is about 0.8 lattice spacing for both systems (in the left lower inset of Fig. 4(b), $\tau$ as a function of $N$ is shown for a few systems up to $N=144$ ). These results clearly illustrate that the ground state is magnetically dis-

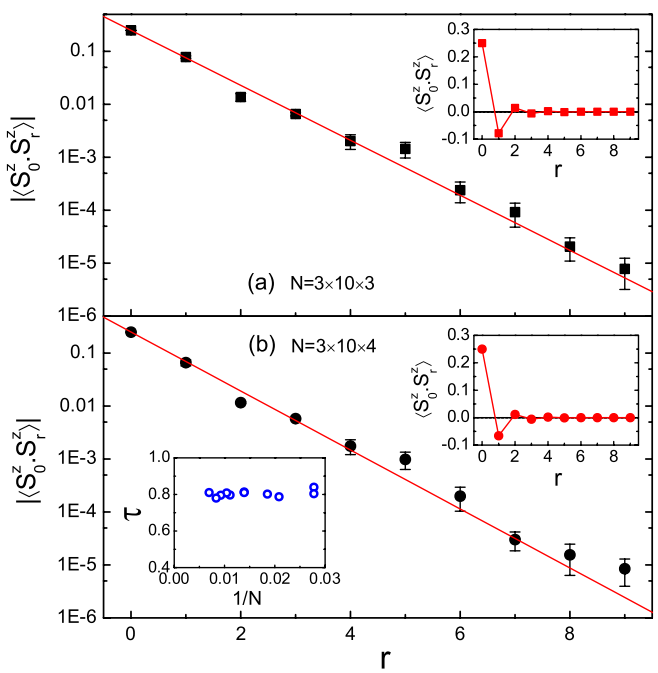

FIG. 4 (color online). The spin-spin correlations $\left|\left\langle S_{0}^{z} S_{r}^{z}\right\rangle\right|$ along the $\mathbf{a}_{1}$ direction with $N=3 \times 10 \times 3$ and $N=3 \times 10 \times 4$. The error bar represents the mean square deviation of all the equivalent sites. The straight line is a fitting to an exponential function $\left|\left\langle S_{0}^{z} S_{r}^{z}\right\rangle\right|=A \exp (-r / \tau)$. The system size dependence of the correlation length $\tau$ is shown in the lower left inset. The spinspin correlations $\left\langle S_{0}^{z} S_{r}^{z}\right\rangle$ are also given in the insets.

ordered with no long-range correlations. Furthermore, $\left\langle S_{0}^{z} S_{r}^{z}\right\rangle$ itself exhibits short-range antiferromagnetic oscillations commensurate with the lattice constant along the $\mathbf{a}_{1}$ direction as shown in the top right inset of Fig. 4. Completely similar results are found for the transverse spin-spin correlation $\left\langle S_{i}^{+} S_{j}^{-}\right\rangle$due to the spin rotational symmetry.

To further characterize the magnetic correlations, we present the static structure factor $S^{z}(\mathbf{q})=$ $\frac{1}{N} \sum_{i j} e^{i \mathbf{q} \cdot\left(\mathbf{r}_{i}-\mathbf{r}_{j}\right)}\left\langle S_{i}^{z} S_{j}^{z}\right\rangle$ in Fig. 5(a) for a system size (a)

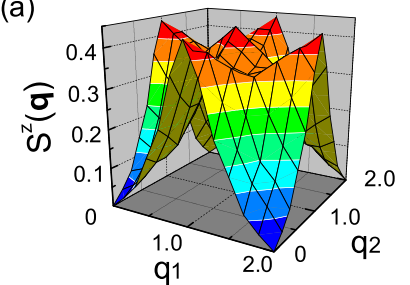

(c)

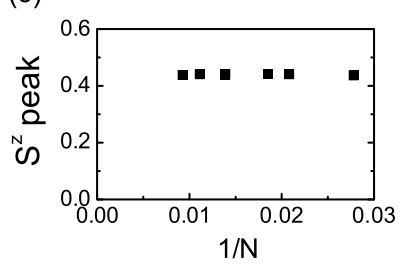

(b)

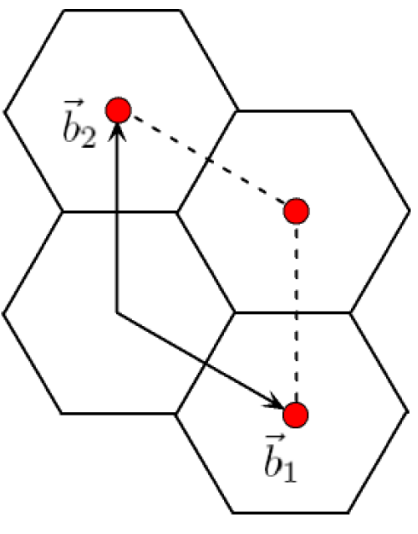

FIG. 5 (color online). (a) The static structure factors $S^{z}(q)$ for the kagome system with $N=3 \times 6 \times 6$ as a function of $\left(q_{1}, q_{2}\right)$ in units of two primitive basis vectors $\left(\mathbf{b}_{1}, \mathbf{b}_{2}\right)$ in the reciprocal lattice. (b) The locations of three peaks in $S^{z}(q)$ marked by solid circles in the extended kagome BZ. (c) The peak values $S^{z}(1,1)$ vs $1 / N$. 
(a)
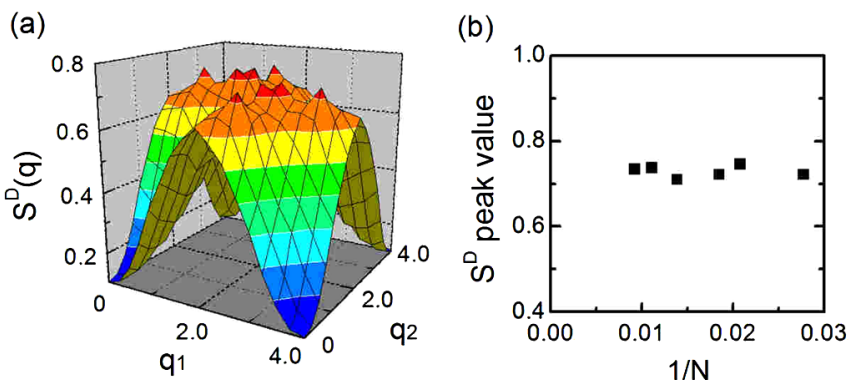

FIG. 6 (color online). The static structure factor $S^{D}$ for the dimer-dimer correlation for a system with $N=3 \times 4 \times 4$ in (a). The peak value of $S^{D}$ vs $1 / N$ is plotted in (b).

$N=3 \times 6 \times 6$, where $\mathbf{q}$ are magnetic wave vectors with components $\left(q_{1}, q_{2}\right)=\left(n_{1} / N_{1}, n_{2} / N_{2}\right)$ in units of two primitive basis vectors $\left(\mathbf{b}_{1}, \mathbf{b}_{2}\right)$ in the reciprocal lattice. We take $0 \leq q_{1}\left(q_{2}\right) \leq 2$ in the extended Brillouin zone (BZ) for a full description of correlations between spins in all three sublattices. $S^{z}(\mathbf{q})$ exhibits small peaks at $q^{*}=$ $(1,0),(0,1)$, and $(1,1)$, as illustrated in the Fig. 5(b). Note that $S^{z}(q)$ is not invariant under reciprocal lattice vector shifts due to 3 -sublattice in a unit cell. It is important to observe that the peaks remain at the same small value $\sim 0.44$ without changing much with increasing system size, as shown in Fig. 5(c). Such weak and sizeindependent peaks are in sharp contrast to the structure factor of a magnetic ordered system in the triangular $\mathrm{HAF}$ model.

Finally, we have also calculated the dimer-dimer correlations $\left\langle\mathbf{S}_{i} \cdot \mathbf{S}_{j} \mathbf{S}_{k} \cdot \mathbf{S}_{l}\right\rangle-\left\langle\mathbf{S}_{i} \cdot \mathbf{S}_{j}\right\rangle\left\langle\mathbf{S}_{k} \cdot \mathbf{S}_{l}\right\rangle(j$ and $l$ are the nearest neighboring sites of $i$ and $k$, respectively) with the corresponding structure factor $S^{D}(\mathbf{q})$ shown in Fig. 6. $S^{D}(\mathbf{q})$ does not have sharp peaks, and more importantly the maximum value of the $S^{D}(\mathbf{q})$ is near constant for all system sizes. These behaviors clearly resemble a spinliquid phase and suggest that VBC is not likely to be realized in such a system. In fact, the overall features of the spin-spin structure factor in Fig. 5 are quite similar to those calculated [22] based on the Gutzwiller projected Dirac spin-liquid state [11], although the spin gap vanishes in the latter. One may thus conjecture the ground state for the kagome HAF be described by an RVB state, which is similar to the projected Dirac spin-liquid state at short ranges. But at long ranges it will have a finite spin triplet gap because of the finite size of spin RVB pairing, and the gapless singlet excitations are Goldstone modes originated from the broken $U(1)$ gauge symmetry due to the RVB condensation, like in a charge-neutral superconductor.
In summary, we have numerically studied the ground state properties and low-lying excitations of the kagome antiferromagnet using the DMRG method. Our results provide strong evidence that the ground state is a spin liquid with only short-range antiferromagnetic correlations without magnetic order or other translational or rotational symmetry breaking. The spin triplet excitation has a gap extrapolated to a finite value in the thermodynamic limit, but the singlet excitation remains gapless. The nature of such a spin-liquid state has been discussed based on the numerical results.

We are grateful for stimulating discussions with L. Balents, Z.C. Gu, T. Li, T. Xiang, and Y. Yu. This work is supported by the NSFC Grant No. 10688401 (H.C.J., Z. Y.W.), the DOE Grant No. DE-FG0206ER46305, and the NSF Grants No. DMR-0605696 and No. DMR-0611562 (H. C. J., D. N. S.).

[1] P. W. Anderson, Mater. Res. Bull. 8, 153 (1973).

[2] D. A. Huse and V. Elser, Phys. Rev. Lett. 60, 2531 (1988).

[3] B. Bernu et al., Phys. Rev. Lett. 69, 2590 (1992).

[4] L. Capriotti et al., Phys. Rev. Lett. 82, 3899 (1999).

[5] F. Wang and A. Vishwanath, Phys. Rev. B 74, 174423 (2006).

[6] P. Lecheminant et al., Phys. Rev. B 56, 2521 (1997).

[7] Ch. Waldtmann et al., Eur. Phys. J. B 2, 501 (1998).

[8] P. W. Leung and Veit Elser, Phys. Rev. B 47, 5459 (1993).

[9] F. Mila, Phys. Rev. Lett. 81, 2356 (1998).

[10] S. Ryu, O. I. Motrunich, J. Alicea, and Matthew P. A. Fisher, Phys. Rev. B 75, 184406 (2007).

[11] Y. Ran et al., Phys. Rev. Lett. 98, 117205 (2007); M. Hermele et al., Phys. Rev. B 77, 224413 (2008).

[12] M. B. Hastings, Phys. Rev. B 63, 014413 (2000).

[13] J. B. Marston and C. Zeng, J. Appl. Phys. 69, 5962 (1991).

[14] R. R. P. Singh and David A. Huse, Phys. Rev. B 76, 180407(R) (2007).

[15] J. S. Helton et al., Phys. Rev. Lett. 98, 107204 (2007).

[16] O. Ofer et al., arXiv:cond-mat/0610540.

[17] P. Mendels et al., Phys. Rev. Lett. 98, 077204 (2007).

[18] A. Olariu et al., Phys. Rev. Lett. 100, 087202 (2008).

[19] M. Rigol and R. R. P. Singh, Phys. Rev. Lett. 98, 207204 (2007).

[20] Steven R. White, Phys. Rev. Lett. 69, 2863 (1992); 77, 3633 (1996).

[21] The correction to the thermodynamic ground state energy for $N=3 \times 6 \times 6$ system is in the order of $10^{-5}$ estimated using series expansion [14].

[22] T. Li (unpublished). 